\title{
Medien- und Telekommunikationspolitik: Unordnung durch Konvergenz - Ordnung durch Mediamatikpolitik
}

\author{
Michael Latzer \\ ITA-ÖAW, Strohgasse 45/5, 1030 Wien, latzer@oeaw.ac.at
}

Wien, Mai 2006

Ende des 20. Jahrhunderts wird ein Kernstück des über viele Jahrzehnte hinweg gelehrten und praktizierten Ordnungsmodells im Kommunikationssektor brüchig: Die technikorientierte Unterteilung in Medien und Telekommunikation, in Individual- und Massenkommunikation, die sich in unterschiedlichen Regulierungsmodellen und in getrennten politischen Zuständigkeiten für Medienpolitik einerseits und Telekommunikationspolitik andererseits manifestiert. Das Internet ist das Symbol der als Konvergenz bezeichneten Disruption in Politik und Analyse, aber gleichzeitig nur die Spitze des Eisberges, auf den das schwer manövrierbare Regulierungssystem aufgelaufen ist. Seither wird an der Schadensbegrenzung und an einem neuen Kurs der Kommunikationspolitik gearbeitet. Das reformierte Ordnungsmodell soll dem durch Konvergenz veränderten Kommunikationssystem, der Mediamatik, gerecht werden.

Der Beitrag zeichnet vorerst die Genese und Grundzüge der traditionellen Ordnungsmodelle der Medien- und Telekommunikationspolitik nach und thematisiert das Verhältnis von Kommunikationswissenschaft und Politik (Abschnitt 1). Anschließend wird die durch Konvergenz verursachte Unordnung in Wirtschaft, Politik und Wissenschaft herausgearbeitet (Abschnitt 2). Die Analyse von Reformvorschlägen und Reformschritten in Reaktion auf den Konvergenztrend verdeutlicht die Grundzüge einer Neuordnung. Sie wird mittels einer integrierten Mediamatikpolitik angestrebt, die den Sektor nicht mehr vertikal nach technologischen Kriterien oder Industriegruppenzugehörigkeit unterteilt (Abschnitt 3). Im Resümee wird auf den möglichen Bedeutungsgewinn kommunikationswissenschaftlicher Forschung für eine integrierte Mediamatikpolitik hingewiesen (Abschnitt 4).

\section{Ordnung durch Abgrenzung: Telekommunikations- und Medienpolitik}

Die national abgeschotteten Kommunikationssektoren des 20. Jahrhunderts sind in ihren Grundzügen durch weitgehend gleichförmige Ordnungsmodelle geprägt, die fundamental zwischen Telekommunikations- und Medienpolitik unterscheiden. Telegrafie und Telefonie, die sich ab der zweiten Hälfte des 19. Jahrhunderts kommerziell etablieren, werden der Telekommunikation zugeordnet, der sich einige Jahrzehnte später kommerziell durchsetzende Rundfunk wird gemeinsam mit der Presse als Mediensektor gefasst. In den Subsektoren Telekommunikation und Medien werden unterschiedliche Technik und getrennte Netze benutzt, es sind unterschiedliche Unternehmen tätig, die politischen Zuständigkeiten sind getrennt, es werden separate Regulierungsorganisationen und gesetzliche Grundlagen geschaffen und auch die zugrunde liegenden Regulierungsmodelle unterscheiden sich voneinander (vgl. Latzer 1997). Für die durch Individualkommunikation geprägte Telekommunikation setzt sich das Common-Carrier-Modell durch, welches auf den Versorgungsauftrag im öffentlichen Interesse fokussiert. Im Medienbereich etablieren sich ein Rundfunkmodell (Public-Trustee-Modell) und ein stärker marktorientiertes Printmodell. KabelTV, das in späterer Folge die Konvergenz der Bereiche vorantreiben wird, nimmt mit einem Mischmodell aus Rundfunk- und Common Carrier-Regulierung eine Sonderposition 
ein. Die Zuteilung $\mathrm{zu}$ den jeweiligen Ordnungsmodellen erfolgt technologie- bzw. industriespezifisch, aufgrund der jeweils benutzten Kommunikationsnetze, die wesentlichen Unterschiede in den Modellen liegen bei der Inhalts- und Marktzutrittsregulierung (vgl. Windahl/McQuail 1993). Dies ist mit unterschiedlichen Regulierungszielen erklärbar, aber auch gleiche Ziele, beispielsweise Vielfalt, werden unterschiedlich angestrebt, im monopolistischen Rundfunksektor mittels Binnenpluralismus und im Printbereich mit Außenpluralismus. Während die traditionelle Rundfunkregulierung stark unter dem Einfluss der sozialen und kulturellen Auswirkungen der transportierten Inhalte sowie der Frequenzknappheit agiert, dominieren im Telekommunikationssektor ökonomische Aspekte im Hinblick auf Infrastrukturleistungen und sicherheitspolitische Überlegungen. Mit der international vorangetriebenen Marktöffnung der Telekommunikation im letzten Drittel des 20. Jahrhunderts rücken ökonomische Kalküle betreffend des Übergangs vom Monopol zum Wettbewerb und damit verbundene Institutionalisierungen von unabhängigen nationalen Regulierungsbehörden in das Zentrum politischer Strategien.

In der Kommunikationswissenschaft spiegelt sich die Trennung in Telekommunikation und Medien wieder, wobei im deutschsprachigen Raum die exklusive Fokussierung auf Medien, auf öffentliche Massenkommunikation, vorherrscht. Telekommunikation wird aus den kommunikationswissenschaftlichen Analysen weitgehend ausgeblendet, so auch bei der politischen Perspektive. „Kommunikationspolitik kann sich vernünftigerweise allein auf öffentliche Kommunikation beziehen, und das bedeutet schwergewichtig auf Medienkommunikation“ (Ronneberger 1992, 195). Eine andere Sichtweise, die einen besseren Blick auf das Konvergenzphänomen erlaubt, versteht Kommunikationspolitik als Summe aus Telekommunikations- und Medienpolitik (vgl. Bentele/Brosius/Jarren 2006). Die Einzigartigkeit der Kommunikationspolitik und gleichzeitig die besondere Komplexität der damit verfolgten Ordnungspolitik liegen in der Kombination aus ökonomischen und sozialen, beziehungsweise kulturellen Zielsetzungen, die damit verfolgt werden. Regulierungsentscheidungen haben sowohl ökonomische als auch soziale Implikationen, die einander überlappen können (vgl. Napoli 2001). Dieser ökonomisch-soziale Doppelcharakter ist im Medienbereich stärker ausgeprägt, spielt aber auch in der Regulierung des Telekommunikationsektors des 20. Jahrhunderts eine bedeutende Rolle, etwa bei Universaldienstauflagen im Telefoniebereich oder bei Beschränkungen des ausländischen Besitzes an Telekommunikationsfirmen aufgrund nationaler Sicherheitskalküle.

Äußerst stabile Marktstrukturen, gekennzeichnet durch Monopole bei Dienst- und Netzangeboten sowie durch Oligopole und nationalen Protektionismus auf der Geräteseite führen zu einem geringen Ausmaß an wissenschaftlicher Politikanalyse des Sektors. Dies ändert sich mit einsetzender Liberalisierung - das wissenschaftliche Interesse an der Kommunikationspolitik verstärkt sich. Rechts-, Wirtschafts-, Kommunikations- und Politikwissenschaft befassen sich seit Ende des 20. Jahrhunderts intensiv mit Medien- und Telekommunikationspolitik, jedoch in verschiedenen disziplinären Zusammensetzungen, in getrennten akademischen Communities, meist auf unterschiedlichen Konferenzen und in verschiedenen Publikationsorganen. Auch hier sind Veränderungen in Folge des Konvergenztrends beobachtbar.

Die Frage nach der Relevanz der Kommunikationswissenschaft für die Formulierung der Kommunikationspolitik wird über Jahrzehnte hinweg mit einheitlichem Tenor beantwortet. Im Konzert der Disziplinen wird ihr nur ein geringer Einfluss zugesprochen (vgl. Noam 1993, Napoli/Gillis 2005). So fassen beispielsweise Reeves \& Baughman (2003) die Ergebnisse ihrer historischen Untersuchung über den Zusammenhang zwischen Kommunikationsforschung und Politik in den USA folgendermaßen zusammen: „,...the 
crucial political decisions have largely ignored mass communication scholarship. Further, government has been more likely to influence research than research to shape policy“. Im Zuge der wechselseitigen Beeinflussung zwischen Politik und Forschung dominiert demnach der oft weniger beachtete Einfluss der Politik auf die Forschung. Dies nicht zuletzt da Topdown-Forschungsförderungsprogramme und deren rigide Richtlinien zunehmend das Forschungsdesign und die behandelten Themen der immer mehr von Drittmittel abhängigen Forschungslandschaft bestimmen. Für den mangelnden Einfluss der kommunikationswissenschaftlichen Forschung auf die Politik wird die geringe Vertretung von Kommunikationswissenschaftern in Regulierungsorganisationen mitverantwortlich gemacht, in denen Juristen, Techniker und Ökonomen das Sagen haben. Des Weiteren wird angeführt, dass es bei kommunikationswissenschaftlichen Ergebnissen zu stärkerem außerdisziplinären Widerspruch komme als bei anderen Disziplinen, und nicht zuletzt sei auch das Engagement von Kommunikationsforschern in öffentlichen kommunikationspolitischen Debatten zu gering. Schießlich bestehen auch Zweifel daran, die durch Liberalisierung und Konvergenz verstärkt notwendige Interdisziplinarität zu gewährleisten. Noam (1993) moniert eine disziplinäre Engstirnigkeit, und Ronneberger (1992) stellt fest, dass der Kommunikationswissenschaft die begriffliche Annäherung und gemeinsame Erklärungsmuster mit anderen Disziplinen fehlen.

\section{Unordnung durch Konvergenz}

\subsection{Konvergenz}

In den Sozialwissenschaften wird der Konvergenzbegriff für die Charakterisierung unterschiedlicher Phänomene herangezogen. In der Politikwissenschaft für die Annäherung von politischen Regimen, insbesondere des westlich-kapitalistischen und östlichrealsozialistischen Systems, und in der Technikforschung wird unter dem Schlagwort NBICKonvergenz (auch ,konvergierende Technologien“) die Annäherung und Verschmelzung von Nano-, Bio- und Informationstechnologien mit Cognitive Sciences diskutiert. In der Kommunikationswissenschaft wird der Konvergenzbegriff zum einen für die Annäherung zwischen öffentlich-rechtlichem und kommerziellen Rundfunkangeboten in dualen Ordnungsmodellen verwendet und zum anderen für das in diesem Beitrag analysierte Verschwimmen der traditionellen Grenzziehungen zwischen Telekommunikation und Massenmedien. Darüber hinaus ist $\mathrm{zu}$ beachten, dass innerhalb der telekommunikationspolitischen Debatte auch die Integration von kabelgebundener und mobiler (Telefon-)Kommunikation als Konvergenz bezeichnet wird. Widersprüchliche Festlegungen gibt es zudem beim Begriff Telekommunikation. Falls Telekommunikation als Kommunikation mittels nachrichtentechnischer Übertragungsverfahren definiert wird (vgl. Scherer 1985), dann umfasst Telekommunikationspolitik auch die Rundfunkpolitik. Dem entgegen steht die hier verwendete Festlegung der Telekommunikations- und Medienpolitik als Bestandteile der Kommunikationspolitik, wobei sich die Analyse auf Regulierungspolitik konzentriert.

Die Konvergenz im Kommunikationssektor wird bereits seit den 1980er Jahren als unabwendbar und wünschenswert diskutiert, früher und intensiver in Telekommunikationsals in Medienkreisen und vorwiegend im Zusammenhang mit angestrebten integrierten Breitbandnetzen und -diensten (vgl. Garnham/Mulgan 1991). Medienvertreter interpretieren den Konvergenztrend eher zurückhaltend, setzen ihn mit Deregulierung und Kommerzialisierung gleich, vermitteln mitunter den Eindruck, dass Konvergenz die 
feindliche Übernahme durch die Telekommunikation darstelle. Die Telekommunikationsbranche steckt bereits vor drei Jahrzehnten große Erwartungen in ISDNBreitbandnetze und Glasfasertechnologie - die sich bis heute nicht erfüllt haben. Nationalstaaten und die Europäische Gemeinschaft entwickeln Strategien zur Förderung konvergenter Breitbandnetze. Mit Telekommunikation und Rundfunk treffen zwei unterschiedliche Welten, zwei Unternehmenskulturen aufeinander. Dementsprechend wirft die OECD (1992) die bezeichnende Frage auf, ob es sich um Konvergenz oder vielmehr um eine Kollision zwischen Telekommunikation und Rundfunk handle, die Europäische Union setzt im Jahr 1997 das Konvergenzthema mit dem Grünbuch zur Konvergenz der Branchen Telekommunikation, Medien und Informationstechnologie und ihren ordnungspolitischen Auswirkungen (Kom97, 623) auf die offizielle politische Agenda. Damit greift die EU, nachdem sie innerhalb eines Jahrzehnts die harmonisierte Liberalisierung der europäischen Telekommunikationssektoren durchgesetzt hatte, ein weiteres brisantes Reformthema auf, das die Liberalisierungsdebatte an Komplexität übertrifft. Auch die Kommunikationswissenschaft beschäftigt sich seit den 1990er Jahren mit den Charakteristika und möglichen Konsequenzen des Konvergenztrends (vgl. Steinfield et al. 1996; Latzer 1997; 1998; Latzer et al. 1999; Bohlin et al. 2000).

Aus analytischer Perspektive vollzieht sich die Konvergenz auf mehreren Ebenen, wobei deren Wechselwirkungen von besonderer Bedeutung sind (vgl. Latzer 1997; Meier 1999; Murdock 2000). Der Technischen Konvergenz kommt dabei eine bedeutende Rolle zu, doch die immer wieder anzutreffende Reduktion darauf, noch dazu verknüpft mit äußerst simplen Erwartungen eines alles integrierenden Einheitmediums, ist verfehlt und irreführend. Technische Konvergenz steht für einen universellen digitalen Kode, für gemeinsame (IP)Protokolle, die auf verschiedenen technologischen (Hybrid)Plattformen (Festnetzkommunikation, Mobilkommunikation, WLAN, Rundfunk, WiMAX ...) verwendet werden. Die Konvergenz schafft ein digitales Baukastensystem, das eine große Flexibilität für innovativ zusammenstellbare Dienste bietet. Dies kann zu diensteintegrierenden Geräten führen wie etwa dem Mobiltelefon, das auch Fernsehen ermöglicht. Konvergenz bringt aber auch und vor allem erhöhte Flexibilität im Angebot, damit steigende Produkt-Vielfalt, da die vormals starre Verknüpfung von Technik und Inhalten (Diensten) aufgelöst wird. Technische Konvergenz fördert auch unternehmerische Konvergenz, d. h. Firmen sind nun in beiden Bereichen tätig, beziehungsweise auch in einem dritten Bereich, dem Internet (Stichwort Triple Play). Dort entstehen zudem, ausgehend vom Kerngeschäft Suchmaschinen (z.B. Google) und elektronischer Handel (z.B. eBay), Konvergenzunternehmen einer neuen Art. Als gesellschaftlich-funktionaler Konvergenz wird erfasst, dass Telekommunikation nun auch zunehmend für den privaten Unterhaltungsbereich und Rundfunk für die Geschäftskommunikation (z.B. unternehmensinternes Business TV) verstärkt verwendet werden. Damit zusammenhängend kommt es zu Verschiebungen, Substitutionen und Kombinationen in der Anwendung von Diensten. Dies wird auch als rezeptive Konvergenz bezeichnet, da es sich um die Veränderung des Nutzungsverhaltens, um eine Konvergenz der Gebrauchsweisen handelt (vgl. Höflich 1999; Hasebrink 2003; Wagner et al. 2004). Schließlich kann eine räumliche Konvergenz festgestellt werden, welche die globalisierende Wirkung vermehrt grenzüberschreitender Dienste und einheitlicher Technik fasst sowie eine regulatorische Konvergenz, die die Abstimmung beziehungsweise Integration der Regulierungssysteme für Medien und Telekommunikation thematisiert.

Das Ergebnis der Konvergenz von Telekommunikation und Rundfunk ist mehr als die Summe der Einzelteile, wie schon der schwer einordbare Bereich der Online-Kommunikation verdeutlicht. Die konzeptive und begriffliche Fassung des Konvergenztrends variiert mit der 
Forschungsperspektive. So wird das Ergebnis der Konvergenz als Multimedia, Crossmedia oder Medienverbund bezeichnet, womit der medienübergreifende Charakter im Angebot und bei der Nutzung betont wird. Aus der für die Kommunikationspolitik relevanten Medienstruktur-Perspektive verändert Konvergenz das techno-soziale, gesellschaftliche Kommunikationssystem in Richtung Mediamatik (vgl. Latzer 1997). Der Computersektor fungiert als Bindeglied zwischen den vormals getrennten Subsektoren Telekommunikation und Medien. Chronologisch betrachtet vollzieht sich die Konvergenz in zwei Schritten. Nach dem mit der Digitalisierung der Telefonie erfolgtem Einzug der Computertechnologie (InforMATIK) in die TELEkommunikation (= TELEMATIK), folgt seit Ende des 20. Jahrhunderts die Konvergenz der ebenfalls digitalisierten (Massen)Medien mit der Telematik (= MEDIAMATIK). Der Prozess erfolgt ko-evolutionär, das wechselseitige Zusammenspiel technischer Innovationen, unternehmerischer Strategien, politisch-rechtlicher Reformen sowie Veränderungen des Rezeptionsverhaltens beziehungsweise der Akzeptanz, bestimmen dessen Richtung und Geschwindigkeit. Das Platzen der Internetblase zur Jahrtausendwende hat den Prozess kurzfristig verlangsamt aber keineswegs gestoppt. Die empirische Evidenz für Konvergenz nimmt im 21. Jahrhundert, v.a. im Zusammenhang mit der Weiterentwicklung von Internet, digitalem Fernsehen, Mobilkommunikation und Next Generation Networks auf allen Ebenen zu.

\subsection{Unordnung}

Konvergenz ist sowohl mit der Globalisierung als auch mit der Liberalisierung kausal verbunden. Digitalisierung im Allgemeinen und Internet im Besonderen verändern die Kostenstrukturen im Kommunikationssektor, ermöglichen und verlangen nach neuen Erlösmodellen und verändern damit die Wettbewerbsbedingungen in konvergenten Mediamatik-Märkten grundlegend. Historisch gesehen ist der erste Konvergenzschritt in Richtung Telematik mit der Liberalisierung des Telekommunikationssektors verknüpft, der grenzüberschreitende Charakter von internetbasierenden Diensten treibt zusätzlich die Globalisierung und dementsprechende Regulierungsprobleme voran.

Die Ordnungsmodelle der Kommunikationspolitik und ihrer wissenschaftlichen Erfassung geraten Ende des 20. Jahrhunderts unter erhöhten Reformdruck, wobei das zentrale Problem darin besteht, dass die Industrie bereits in die Mediamatik-Ära eingetreten ist, während Politik und wissenschaftliche Analyse nach wie vor mit in Medien und Telekommunikation getrennten Institutionen und Regulierungssystemen agieren. Die digitale Destabilisierung der Ordnungssysteme geht von zunehmend obsoleten Grenzziehungen aus, die seit Jahrzehnten als zentrale Unterscheidungskriterien zwischen Medien und Telekommunikation und damit auch für die Zuteilung $\mathrm{zu}$ unterschiedlichen Regulierungsmodellen dienen: technologisch orientierte, vertikale Sektorabgrenzungen, die Unterscheidung in Massen- und Individualkommunikation, in öffentliche und private Kommunikation sowie die strikte Trennung von Sender (Anbieter) und Empfänger (Nachfrager).

Dies sind nicht bloß akademische Probleme der Kommunikationswissenschaft, sondern sie haben realwirtschaftliche und realpolitische Auswirkungen. Die Rechtsunsicherheit steigt an, die Planungssicherheit der Unternehmen sinkt und das Investitionsrisiko erhöht sich. In Summe kann dadurch die Entfaltung des für die Informationsgesellschaft als zentral erachteten Sektors (Stichwort Lissabon-Strategie der EU) empfindlich gehemmt werden. Zur Rechtsunsicherheit trägt die steigende politische Willkür bei der Kategorisierung neuer Dienste bei. So wird Internet-Telefonie nicht als Telefonie 
eingestuft und $W e b-T V$ nicht als Fernsehen, um damit verbundene regulatorische Auflagen (Universaldienst, Zugangsgebühren, Interconnection, Inhaltsregulierungen etc.) zu vermeiden, die deren Verbreitung hemmen könnten (vgl. Dong 2006). Bei Breitbandangeboten wird einerseits zu deren Förderung darauf geachtet, dass nicht das Common-Carrier-Modell und damit dessen regulatorische Auflagen zur Anwendung kommen. Andererseits wird diskutiert, ob Common-Carrier-Modelle aufgrund der darin fehlenden Inhaltsregulierung für Breitbandnetze ausreichen. Bei Triple-Play Anbietern stellt sich die Frage, ob deren relevanter Markt nun der für Breitband ist. Rechtsunsicherheit ergibt sich auch bei Fragen der Verantwortlichkeit für Inhalte, ob etwa Internet-Provider wie Telefonie-Anbieter Common Carrier und damit nicht - wie Herausgeber von Massenmedien - für den Inhalt verantwortlich sind. Schließlich führt auch die umstrittene Frage ob Inhalte von Webseiten öffentlich oder privat sind $\mathrm{zu}$ Rechtsunsicherheiten, etwa ob regulatorische Auflagen des NSWiederbetätigungsgesetzes anwendbar sind. Die Rechtsunsicherheiten steigen auch aufgrund der räumlichen Konvergenz an, die gleichsam als Globalisierung diskutiert wird. Die Kombination von vorwiegend nationalen Regulierungen und transnationalen Diensten führt nicht nur zur umstrittenen Frage, ob die Regulierung des Herkunfts- oder des Empfängerlandes anzuwenden ist, sondern auch zu vermehrten Umgehungsmöglichkeiten nationalen Rechts, zur verstärkten Notwendigkeit trans- und supranationaler Regulierungen sowie zur vermehrten Selbst- und Ko-Regulierung. Insgesamt verursachen Rechtsunsicherheiten aus ökonomischer Sicht erhebliche Transaktionskostenerhöhungen, etwa bei Rechtsstreitigkeiten, aber auch in Form von Suchkosten für Dienstanbieter und für Konsumenten. Unordnung entsteht auch, da demokratiepolitisch motivierte Regelungen für den Rundfunk, etwa bei der Wahlberichterstattung, durch internetbasierende Dienste legal umgangen werden können.

Neben den oben skizzierten Problemen aufgrund obsoleter Grenzziehungen entstehen durch den Konvergenztrend auch neue Regulierungsaufgaben, beziehungsweise gewinnen sie an Bedeutung. Etwa der Schutz geistigen Eigentums, die Besteuerung von Internethandel, die Regulierung von Kryptographie und von Domainnamen-Systemen.

Insgesamt kommt es zu einer politischen Steuerungskrise im Kommunikationssektor. Die Steuerbarkeit des Sektors mittels Kommunikationspolitik, vor allem wenn es um die Erreichung mittel- und langfristiger Ziele geht, wird zunehmend bezweifelt. Dies u. a. auch, da die Komplexität des zu steuernden Sektors durch Liberalisierung und Konvergenz ansteigt, da die Anzahl der zu berücksichtigenden Akteure und deren Verknüpfungen massiv zunehmen (vgl. Verhoest 2005).

Die öffentlichen Regulierungsziele im Medien- und Telekommunikationsbereich bleiben gleich, sie umfassen nach wie vor einen Mix aus ökonomischen und sozialen Aspekten. Die Zielerreichung mittels der traditionellen Ordnungsmodelle verringert sich jedoch im konvergenten Mediamatiksektor. Dazu tragen nicht nur obsolete regulatorische Grenzziehungen bei, sondern auch die erhöhte Innovationsgeschwindigkeit bei Technik und Diensten, der Wandel der Industriestruktur und des Benutzerverhaltens sowie die steigende Zahl relevanter Akteure.

Als Auswirkung der Konvergenz wird die verstärkte Ökonomisierung des Medienbereiches erwartet, beziehungsweise die Zurückdrängung sozial motivierter Regulierungen befürchtet. Als Indiz dafür gilt die Stärkung der Rolle des allgemeinen Wettbewerbsrechts im konvergenten Kommunikationssektor auf Kosten sektorspezifischer Regulierungen (vgl. Just 2005). Sie findet im Telekommunikationssektor statt, etwa im neuen Rechtsrahmen der EU für elektronische Kommunikation, und wird im Zuge der 
Konvergenzdebatte auch als Variante einer vereinheitlichenden, integrativen Strategie für den Mediensektor diskutiert.

$\mathrm{Zu}$ berücksichtigen ist aber auch, dass durch die Konvergenz jene Besonderheiten der Kommunikationspolitik gestärkt werden, die durch die Mischung aus sozialen/kulturellen und ökonomischen Regulierungen entstehen (vgl. Napoli 2001). Diese sind nun auch im Telekommunikationsbereich in einzelnen Entscheidungen verstärkt $\mathrm{zu}$ beachten. Beispielsweise sind bei Veränderungen der Besitzstrukturen von Telekommunikationsunternehmen, die nun TV-Dienste im Portfolio haben, auch die sozialen/kulturellen Auswirkungen zu berücksichtigen. Auch bei der Frequenzpolitik vermischen sich zunehmend die ökonomischen Ziele des effizienten FrequenzspektrumManagements mit sozialen Zielsetzungen, wie Vielfalt und nationale Identitätsstiftung. Plattformen, die die ganze Palette an konvergenten Diensten erlauben und Endgeräte, die die ganze Palette an Diensten ermöglichen, führen des Weiteren dazu, dass der ökonomische Wert der Frequenzen ansteigt. Die Verteilung der „digitalen Dividende“, des durch die Digitalisierung des Fernsehens frei werdenden Spektrums, das nicht an die spezifische Verwendung für weitere TV-Kanäle gebunden ist, gewinnt unter diesem Aspekt an Bedeutung. Die traditionell unterschiedliche Lizenzpolitik für Telekommunikation und Rundfunk greift nicht mehr, die Kriterien und damit verbundenen Auflagen erfüllen oft nicht mehr ihren ursprünglichen Zweck. Insgesamt kann die fragmentierte Rundfunk- und Telekommunikationsregulierung des traditionellen Ordnungsmodells, mit unterschiedlichen Auflagen je nach verwendeter Übertragungstechnik, beziehungsweise mit unterschiedlichen Vergabemechanismen und Preisstrukturen je nach Industriegruppe, für ein konvergentes Umfeld weder ein effizientes Ressourcenmanagement noch die Erreichung sozialer und kultureller Ziele gewährleisten (vgl. OECD 2003). Auch ein weiterer Eckpfeiler der Kommunikationspolitik, die Universaldienstpolitik, ist unter Konvergenzbedingungen integrativ zu gestalten, wobei ökonomische und soziale Aspekte abzuwägen sind (vgl. Simpson 2004). Bei den Universaldienstzielen, auch jenen des öffentlich-rechtlichen Auftrags, ist im konvergenten Mediamatiksektor neu zu hinterfragen, mit welchen Diensten und auf welchen Plattformen sie am besten erreichbar sind (vgl. Latzer 2000).

Der Konvergenztrend stellt mit den eingangs genannten Abgrenzungsproblemen nicht nur die Fundamente der medien- und telekommunikationspolitischen Praxis, sondern auch der Kommunikationswissenschaft in Frage. Konvergenz findet langsam, aber beständig eine adäquate Berücksichtigung in den verschiedenen Teilgebieten, den Bindestrich-Fächern der kommunikationswissenschaftlichen Disziplin. Dies passiert nicht nur in der Medienpolitik, ökonomie und dem Medienmanagement, sondern auch in der Medientheorie, der Medienpädagogik, der Journalismus- und Rezeptionsforschung. Darüber hinaus befinden sich neue Teilgebiete in verschiedenen Stadien der Etablierung, wie Online-Kommunikation und Medieninformatik (vgl. Bentele/Brosius/Jarren 2003). Angesichts des Konvergenztrends wird die traditionelle Abgrenzung des Untersuchungsgegenstandes der Kommunikationswissenschaft kritisiert und dessen Ausdehnung auf Telekommunikation empfohlen. Vorgeschlagen werden damit zusammenhängend auch Revisionen der Medienund Rundfunkbegriffe, der Trennung von Sender und Empfänger sowie der Form der Unterteilung in öffentliche und private Kommunikation (vgl. Latzer et al. 1999; Neverla 2001).

Für die Kommunikationswissenschaft sind derart motivierte Debatten und Herausforderungen nicht ungewöhnlich. Mit Saxer (2006) habe die Kommunikationswissenschaft ihrem expansiven und dynamischen Gegenstand $\mathrm{zu}$ folgen, 
handle sich dabei Grenz- und Kompetenzprobleme ein und müsse inter- und transdisziplinären Optimierungsregeln genügen. Die Herausforderung einer Nachadjustierung des Untersuchungsgegenstandes als Reaktion auf „Neue Medien“ ist für die Disziplin ein bekanntes Phänomen. So ist die als Zeitungskunde vor rund einem Jahrhundert etablierte Disziplin bereits in Reaktion auf Radio und Rundfunk zur Publizistik- bzw. Kommunikationswissenschaft mutiert. Die von Saxer betonte Gefahr der Kompetenzüberdehnung ist im Fall der Hinzunahme von Telekommunikation und Internet größer als sie bei der Hinzunahme von Rundfunk gewesen war. Sie ist jedoch mit der im Gegenzug steigenden Gefahr des Realitäts- und Bedeutungsverlustes kommunikationswissenschaftlicher Forschung für das Verständnis aktueller Entwicklungen der Informationsgesellschaft sowie für die Politikformulierung des konvergenten Kommunikationssektors abzuwägen. Denn selbst Strategien für massenmediale Kernbereiche wie Presse und Rundfunk, von der Wettbewerbspolitik und Marktmachtkontrolle über die Frequenzpolitik bis hin zur Presseförderung und dem Jugendmedienschutz, bleiben ohne gleichzeitige Berücksichtung der Telekommunikations- und Internetentwicklungen, ohne den integrativen Blick auf das konvergente Kommunikationssystem der Mediamatik realitätsfern.

\section{Neuordnung}

Reformvorschläge in Reaktion auf den Konvergenztrend und die dadurch mitverursachte Steuerungskrise kommen aus Wissenschaft und Politik. Sie zielen mittels eines evolutionären Prozesses auf ein verändertes Ordnungsmodell ab, auf eine integrierte Mediamatikpolitik (vgl. Latzer 1997; 1998), beziehungsweise auf eine gemeinsame Regulierung von elektronischer Kommunikation (vgl. OECD 2003) und sie arbeiten die nationalen Besonderheiten von regulatorischen Reformen heraus (vgl. Hoffmann-Riehm/Schulz/Held 2000; Roßnagel 2005).

Das Kernstück des neuen Ordnungsmodells wird oft vage als Konvergenz der Regulierung bezeichnet. Wie bei technischer Konvergenz so wäre es auch hier irreführend, sich darunter eine detaillierte Einheitslösung für sämtliche Netze, Dienste und Inhalte des konvergenten Mediamatikbereiches vorzustellen. Eine differenzierte Betrachtung von Reformvorschlägen und Umsetzungsschritten lässt die Richtung und Grundzüge einer als notwendig erachteten Neuordnung erkennen. Wie schon beim traditionellen Ordnungsmodell, so ist auch für eine zukünftig integrierte Mediamatikpolitik international ein in groben Zügen einheitliches Politikmuster zu erwarten.

Es wird zeitliche und inhaltliche Abweichungen geben, im Detail werden und sollen sich die institutionellen Reformen in den einzelnen Staaten unterscheiden, und sie sind auch länderspezifisch zu beurteilen. Denn die Ausgangsbedingungen, der politisch-rechtliche Rahmen, die Interessenskonstellationen und Kräfteverhältnisse, der Grad an bereits bestehender Integration von Telekommunikations- und Medienpolitik und auch die Geschwindigkeit der Konvergenz variieren beträchtlich zwischen den Staaten. So macht es einen wesentlichen Unterschied, ob die politischen Kompetenzen für Telekommunikation und Rundfunk wie in Deutschland auf Bund (Telekommunikation) und Länder (Medien) verteilt sind, auf verschiedene Ministerien wie in Österreich, oder wie in Japan in einem Ministerium zusammengefasst sind. Pfadabhängigkeiten der Entwicklungen bedürfen der besonderen Berücksichtigung, gleich wie Interessenpositionen und damit zusammenhängende Machtverteilungen, die wie in Deutschland dazu führen können, dass regulatorische Kategorisierungen von Onlinediensten nicht sachpolitisch sondern als politischer Kompromiss zwischen Bund und Ländern festgelegt werden (vgl. Roßnagel 2005). Das 
institutionelle Beharrungsvermögen, die Trägheit des Systems ist generell groß, insbesondere da jede organisatorische Veränderung auch mit Machtgewinnen und -verlusten verbunden ist.

Trotz der zu erwartenden Vielfalt an nationalen Detaillösungen lassen sich aus einer institutionalistischen Perspektive einige Entwicklungslinien ableiten, beziehungsweise können diese aus rezenten Entwicklungen abgelesen werden. Dafür werden nachfolgend Analysen aus Wissenschaft und Politik sowie Reformschritte herangezogen, die bereits von einzelnen Nationalstaaten oder transnationalen Akteuren gesetzt wurden.

\section{Integrierte Strategie - Integration der politischen Zuständigkeiten: Alles im Blick}

Der Reformbedarf und auch die Reformschritte setzen sowohl bei den politischen Strategien als auch bei den konkreten Regulierungen an. Im traditionellen Ordnungsmodell der Kommunikationspolitik sind Strategieentwicklung (Regulierungsrahmen) und Implementierung der Regulierung meist gemeinsam in den jeweils zuständigen Ministerien angesiedelt. Im Zuge der Liberalisierung kommt es zur Trennung und Auslagerung der Telekommunikationsregulierung aus der öffentlichen Verwaltung in unabhängige Regulierungsorganisationen. Während noch vor einem Jahrzehnt beinahe alle europäischen Telekommunikationsregulatoren Teil der öffentlichen Verwaltung waren, so ist dies heute in keinem EU-Mitgliedsland mehr der Fall. In den OECD-Ländern ist einzig in Japan und Korea die strategische und regulatorische Kompetenz in einem Ministerium konzentriert (vgl. OECD 2005). Die politische Strategieentwicklung bleibt im Zuständigkeitsbereich der öffentlichen Verwaltung, beziehungsweise der Parlamente.

Bei einer integrierten Mediamatikpolitik lautet die Zielsetzung, den gesamten Kommunikationssektor bei der Strategieentwicklung im Blick zu haben, wobei nicht nur der elektronische (Rundfunk, Telekommunikation, Online), sondern auch der nicht-elektronische Medienbereich einzubeziehen ist. Aus institutionellem Blickwinkel erleichtern gebündelte politische Zuständigkeiten die Erstellung integrierter Strategien. Je nach nationaler Ausgangslage kann es dafür notwendig sein, die politischen Kompetenzen für Telekommunikation und Medien mittels institutioneller Reformen zusammenzuführen, und zwar auf der Ebene der Ministerien oder auch im Parlament, wo Telekommunikations- und Medienangelegenheiten oft in unterschiedlichen Ausschüssen behandelt werden.

Ein Beispiel für die Integration auf politisch-strategischer Ebene liefert die EUKommission. Die vormals auf die Generaldirektionen XIII (Telekommunikation) und X (Medien) aufgeteilten Kompetenzen sind seit 2004 in der Generaldirektion Informationsgesellschaft und Medien zusammengefasst. Die Konvergenz ist auch ein Schwerpunktthema der umfassenden Strategie i2010: Europäische Informationsgesellschaft 2010. Sie zielt darauf ab, auch eine Konvergenz von Politik und Technik herzustellen. Sämtliche politische Instrumente der Gemeinschaft sollen modernisiert und eingesetzt werden, um die digitale Wirtschaft zu fördern.

\section{Integrierte Aufsichtsstrukturen - horizontale Konvergenzregulatoren: Alles unter einem Dach}

Nicht nur die Strategieentwicklung bzw. die Schaffung des Regulierungsrahmens, sondern auch die davon getrennte Implementation der Regulierung soll von einer Integration profitieren. Bei der im neuen Ordnungsmodell angestrebten Integration der Regulierung ist zwischen dem Integrationsgrad der Regulierungsorganisationen/Aufsichtsstrukturen und jener der Regulierungsmodelle, bzw. -Inhalte zu unterscheiden. Bis zur Konvergenz sind 
Regulierungsorganisationen zumeist vertikal in jene für Telekommunikation und Rundfunk aufgeteilt, oft gibt es noch weitere Unterteilungen, etwa bei der Rundfunkregulierung in organisatorisch getrennte Aufsichtsorgane für Netze, Frequenzen und Inhalte. In Folge der Konvergenz sind im letzten Jahrzehnt Reformen in Richtung organisatorisch integrierter Konvergenzregulatoren zu beobachten (vgl. OECD 2005; Wu 2004). So ersetzt beispielsweise in Großbritannien seit dem Jahr 2003 das Office of Communications (OFCOM) fünf Aufsichtsorgane und in Australien entstand 2005 die Australian Communications and Media Authority (ACMA) aus der Zusammenlegung der Rundfunk- mit der Kommunikationsregulierungsbehörde. Insgesamt gibt es in rund einem Drittel der OECDLänder bereits Konvergenzregulatoren mit horizontal integrierten Zuständigkeiten für Rundfunk und Telekommunikation und auch in Nicht-OECD Ländern vollzieht sich dieser Entwicklungstrend. Eine weniger weitreichende Integrationsvariante wurde in Österreich gewählt, wo im Jahr 2001 zwar die Geschäftsstellen der Regulierungsbehörden in der Rundfunk \& Telekom Regulierungs-GmbH (mit zwei Fachbereichen und Geschäftsführern) zusammengeführt wurden, die Regulatoren für Rundfunk und Telekommunikation aber getrennt blieben.

Im Detail unterscheiden sich die horizontalen Konvergenzregulatoren, etwa bei den Zuständigkeiten für das Frequenzspektrum, für den Printsektor oder für die Wettbewerbspolitik. In der politischen Praxis ist das Verhältnis des sektorspezifischen Regulators zur allgemeinen Wettbewerbsbehörde unterschiedlich geregelt. So ist der britische Konvergenzregulator OFCOM gemeinsam mit der allgemeinen Wettbewerbsbehörde für die Anwendung des Wettbewerbsrechts zuständig, während in Australien die allgemeine Wettbewerbsbehörde mit sektorspezifischen Rechten für den Telekommunikationssektor ausgestattet wurde. In etlichen Ländern gibt es eigene Abkommen bezüglich der Kompetenzverteilung (vgl. OECD 2003).

Mit dem Grad der Integration variieren auch die erwarteten Vorteile. Selbst mit einer auf die Dachkonstruktion beschränkten Integration lassen sich Effektivitäts- und Effizienzgewinne, nicht zuletzt durch Synergieeffekte und Transaktionskostenersparnisse erzielen. Der Gefahr einer zu hohen Machtkonzentration eines Konvergenzregulators kann mittels institutioneller Vorkehrungen wie etwa Transparenzauflagen institutionell entgegnet werden.

\section{Technikneutrale, funktionale Taxonomie - Übertragungs- und Inhaltsregulierung: Nicht alles über einen Kamm scheren}

Alles im Blick und unter einem Dach zu haben, heißt nicht, dass alles über einen Kamm geschert wird. Die Konvergenz verlangt jedoch nach einer neuen Taxonomie, die sich von der bisherigen Unterteilung entsprechend der verwendeten Technik oder nach Industriegruppen wegbewegt. Denn Technik/Netze und Inhalte/Dienste sind aufgrund der Konvergenz bekanntlich entkoppelt. Die neuen Klassifikationskriterien sind funktional, aktivitätsbezogen und technikneutral. Demgemäß zeichnet sich eine Unterteilung in Übertragungsregulierung und Inhaltsregulierung ab. Damit soll gleichsam eine Trennung in ökonomische und soziale/kulturelle Regulierung vollzogen werden, wobei eine strikte Abgrenzung unmöglich ist, da Entscheidungen im Übertragungsbereich nicht nur ökonomische sondern auch soziale und kulturelle Auswirkungen haben, sich beispielsweise Veränderungen der Gatekeeper auch auf die Inhalte auswirken.

Die Regulierung der Übertragung auf unterschiedlichen technischen Plattformen erfolgt im neuen Ordnungsmodell einheitlich, während bei den Inhalten, abhängig von den 
erwarteten Auswirkungen keine einheitliche Regulierung zur Anwendung kommt. Für die Organisationsstruktur von Konvergenzregulatoren könnte dies bedeuten, dass auch eine organisatorische Unterteilung in Übertragungs- und Inhaltsregulierung erfolgt, wobei das gemeinsame organisatorische Dach eine bessere Berücksichtigung der Wechselwirkungen gewährleistet. Frequenzvergabe und Universaldienstpolitik wären im neuen Ordnungsmodell eher der Übertragungs- als der Inhaltsregulierung zuzuordnen.

In Großbritannien wurde nach jahrelangen Konsultationen - auch dazu, ob ein oder zwei Regulatoren vorteilhaft wären - mit OFCOM ein Konvergenzregulator geschaffen, der sowohl für die Regulierung der Übertragung als auch der Inhalte verantwortlich ist. Unter dem Dach der bereits seit den 1930er Jahren gemeinsam für Telekommunikation und Rundfunk verantwortlichem US-amerikanischen Federal Communications Commission (FCC) gibt es - entsprechend dem traditionellen Schema - nach Industriegruppen in Telekommunikation, Rundfunk und KabelTV unterteilte Organisationseinheiten. Auch das wird unter Konvergenzbedingungen als ineffizient kritisiert und es werden Reformen in Richtung einer funktional orientierten Organisationsstruktur vorgeschlagen (vgl. GarciaMurillo/ MacInnes 2001)

\section{Integrierter Rechtsrahmen und Gesetze}

Der integriert-strategische Blick und die organisatorisch integrierten Konvergenzregulatoren führen auch vermehrt zu integrierten Rechtsrahmen beziehungsweise $\mathrm{zu}$ integrierten Gesetzen, in denen Telekommunikation, Rundfunk und Online-Kommunikation geregelt werden. In den USA wird mit dem Telecommunications Act 1996 ein Integrationsschritt gesetzt. Frühere Pläne eines eigenen Regulierungskapitels für konvergente Dienste wurden damit aufgegeben. Auch die $E U$ reagiert auf den Konvergenztrend. Der seit 2003 gültige Rechtsrahmen für elektronische Kommunikation, der technikneutral formuliert und bereits 2006 einem Überprüfungs-Prozess unterzogen wird, bringt eine Integration und Vereinheitlichung der Infrastruktur-Regulierungen für elektronische Kommunikation, die auf verschiedenen technischen Plattformen stattfindet.

Der in einem ersten regulatorischen Konvergenzschritt 2002 geschaffene Rechtsrahmen für die Infrastrukturregulierung setzt sich aus sechs Richtlinien des Europäischen Parlaments und Rates sowie einer Entscheidung zusammen. Damit wird die Übertragung von Rundfunk-, Telekommunikations- und Onlinediensten integriert geregelt. In einem zweiten Schritt wird die Inhaltsregulierung dem Konvergenztrend angepasst. Dies soll mittels der Revision der Fernsehrichtlinie erfolgen. Die Ende 2005 als Draft zur Diskussion gestellte audiovisuelle Mediendienste Richtlinie sprengt, wie schon der veränderte Name verdeutlicht, die Fixierung auf Fernsehen und soll konvergenzgerechte europäische Standards für die Inhaltsregulierung setzen.

In den Nationalstaaten überwiegen, auch dort wo bereits integrierte Konvergenzregulatoren eingerichtet wurden, getrennte Gesetze. $\mathrm{Zu}$ den Ausnahmen zählt Großbritannien, wo mit dem Communications Act 2003 ein gemeinsames Gesetz geschaffen wurde. Deutschland ist ein Beispiel dafür, wo ein integrierter Rechtsrahmen zwar sachpolitisch als angebracht erkannt, aber machtpolitisch als nicht umsetzbar eingestuft wird. Die Reformen in Reaktion auf die Konvergenz setzen folglich, wenn auch ohne Gesamtkonzept, bei einer konvergenzadäquaten Reform von Teilgebieten, der Vereinheitlichung des Jugendmedien- und Datenschutzes für verschiedene Kommunikationsplattformen an (vgl. Roßnagel 2005). 


\section{Alternative Regulierungsformen: Vom Government zur Governance}

Die Regulierung, das Setzen von Normen, deren Implementierung und Sanktionierung, wird nicht nur mittels nationaler Gesetze und zentraler staatlicher Regulierung vollzogen. Der Konvergenztrend treibt die vertikale und horizontale Erweiterung des klassischen Government in Richtung Governance voran. Vertikal erweitert kommt es vermehrt zu einer Mehrebenenregulierung im Mediamatiksektor, horizontal erweitert zur stärkeren Einbindung von privaten Akteuren in den Regulierungsprozess. Bei der Anwendung von Selbst- und KoRegulierung (alternativer Regulierungsformen) werden zumindest Teile des Regulierungsprozesses (Normsetzung, Vollzug, Sanktionierung) privaten Akteuren überantwortet, die Rolle des Staates verändert sich im Vergleich zum traditionellen Ordnungsmodell. Die Vorteile der Selbst- und Ko-Regulierung gegenüber klassischer staatlicher Regulierung können bei den durch Konvergenz geschaffenen Bedingungen: grenzüberschreitender Charakter der Dienste, rascher technologischer Wandel, zunehmende Anzahl der Akteure, gut genutzt werden. Die Anwendung alternativer, teils innovativer Regulierungsformen nimmt in allen Segmenten zu, insbesondere bei internetbasierenden Diensten, wobei die Palette von der Standardisierung über den Konsumentenschutz und der Domainnamen-Verwaltung bis hin zum Jugendmedienschutz reicht (vgl. Latzer et al. 2002; Schulz/Held 2004).

\section{Resümee}

Politik und Forschung hinken den konvergenzbedingten Veränderungen im Kommunikationssektor hinterher. Das traditionelle Politikmodell mit seiner fundamentalen Trennung in Telekommunikation und Massenmedien, die $\mathrm{ihr} \mathrm{zu}$ Grunde liegenden Unterscheidungsmerkmale und die darauf aufbauenden Aufsichtsstrukturen und Regulierungsrahmen sind zunehmend defizitär, hemmen die Entwicklung des Sektors und mindern die Erreichung von Regulierungszielen. Kombiniert mit einer Komplexitätssteigerung durch zusätzliche Akteure und Regulierungsebenen sinkt bei gleich bleibenden Zielen nicht nur deren Erreichung, sondern auch die Steuerungsfähigkeit im konvergenten Mediamatiksektor. Sowohl die Politik als auch die Wissenschaft reagieren auf den zwar langsamer als erwarteten, jedoch ungebrochenen Konvergenztrend. Trotz aller notwendigen Unterschiede in den einzelnen nationalen Strategien, die nicht zuletzt der Pfadabhängigkeit und spezifischen Interessenkonstellationen geschuldet sind, zeichnet sich ein grobes Muster der Neuordnung für eine integrierte Mediamatikpolitik ab. Dazu trägt auch die durch Konvergenz gleichsam vorangetriebene Globalisierung bei.

Bei der Strategieentwicklung gilt es nun den gesamten Kommunikationssektor gleichzeitig im Blick zu haben, bei der Aufsichtsstruktur geht der Trend in Richtung eines gemeinsamen Daches, wenn auch bei der Regulierung unter diesem Dach nicht alles über einen Kamm geschert werden soll. Anders als bisher wird nicht mehr auf Grund der verwendeten Netztechnik, sondern nach funktionalen, anwendungsabhängigen und technikneutralen Kriterien unterteilt, in eine einheitliche Übertragungsregulierung sowie eine wirkungsabhängig differenzierte Inhaltsregulierung. Ebenso werden die entsprechend veränderten gesetzlichen Grundlagen tendenziell integriert und es gelangen auch vermehrt alternative Regulierungsformen, insbesondere nicht mehr im Detail gesetzlich fixierte Regulierungen unter verstärkter Einbindung privater Akteure, zur Anwendung. Der rasche techno-ökonomische Wandel führt überdies zu dynamischen Ordnungsrahmen, in deren Konzeption bereits periodisch zu erfolgende Reviews eingeplant werden. 
Mit der Konvergenz nimmt nicht nur die Ökonomisierung des konvergenten Sektors $\mathrm{zu}$, sondern auch die oft übersehene Verschränkung sozialer und ökonomischer Implikationen gewinnt im Mediamatiksektor an Bedeutung. In Kombination mit einer verstärkt anwendungs- und wirkungsabhängigen Regulierung im neuen Ordnungsmodell könnte die über lange Zeit von der Politik eher verschmähte kommunikationswissenschaftliche Forschung deutlich an Relevanz und Einfluss in der Politikgestaltung gewinnen. Denn die Politik benötigt als Input für eine integrierte Mediamatikpolitik neben detaillierten institutionalistischen Untersuchungen (Aufsichtsstrukturen, Regulierungsformen und instrumente) u.a. Einschätzungen über (veränderte) Nutzung und Wirkungen des gesamten Anwendungsspektrums. Kommunikationswissenschaftliche Kernkompetenz könnte nicht nur für die wirkungsabhängige Inhaltsregulierung an Bedeutung gewinnen, sondern auch für andere Politikfelder wie etwa Marktmachtkontrolle und die Regulierung von Besitzstrukturen, wo im neuen Ordnungsmodell Ergebnisse der Rezeptions- und Wirkungsforschung ebenfalls höhere Relevanz erlangen (vgl. Napoli/Gillis 2005). Für die Wahl der technikneutralen Regulierung ist beispielsweise von Interesse, inwieweit sich Nutzungsmuster verändern, ob und wie sich etwa die soziale Wirkung von terrestrischem Fernsehen im konvergenten Diensteumfeld verringert. Um eine prominente Rolle im Konzert der Disziplinen zu spielen, ist ein verstärktes Einbringen der Kommunikationswissenschaft in die Fragestellungen gefordert, aber auch die Einsicht, dass eine zeitgemäße Struktur- und Organisationsforschung, aber auch die Nutzungs- und Wirkungsforschung, also Kernbereiche der Kommunikationswissenschaft, nach der systematischen Ausdehnung des klassischen Untersuchungsgegenstandes verlangen. Eine Kommunikationswissenschaft, die auch hier ihrem Anspruch als Integrationswissenschaft gerecht wird, kann zur interdisziplinären Analyse der Kommunikationspolitik wesentlich beitragen. Mit der Stärkung entsprechender (empirischer) Forschung, die sich bislang auf Teilfragen, etwa die Zusammenhänge von Fernseh- und Internetrezeption konzentriert, würde nicht zuletzt auch dem Plädoyer Ulrich Saxers (1999) für eine wissenschaftliche Medienpolitik entsprochen werden.

\section{Literaturverzeichnis}

Baldwin, Thomas. F. / McVoy, Stevens D. / Steinfield, Charles (1996): Convergence, Integrating Media, Information and Communication. London.

Bentele, Günter/ Brosius, Hans-Bernd/ Jarren, Otfried (2006): Lexikon Kommunikations- und Medienwissenschaft. Wiesbaden.

Bentele, Günter/ Brosius, Hans-Bernd / Jarren, Otfried (Hrsg.) (2003): Öffentliche Kommunikation. Wiesbaden.

Bohlin, Eric / Brodin, Karolina / Lundgren, Anders / Thorngren, Bertil (Hrsg.) (2000): Convergence in Communications and Beyond. Amsterdam.

Dong, Hee Shin (2006): VoIP: A debate over information service or telephone application in US: A new perspective in convergence era. Working Paper, School of Information Sciences and Technology, Pennsylvania State University, Reading.

Garcia-Murillo, Martha A. / MacInnes, Ian (2001): FCC organizational structure and regulatory convergence. In: Telecommunications Policy, 25 (6), S. 431-452.

Garnham, Nicholas/ Mulgan, Geoff (1991): Broadband and the barriers to convergence in the European Community. In: Telecommunications Policy, 15 (3), S. 182-194. 
Hasebrink, Uwe (2003): Konvergenz aus Nutzerperspektive. Zur Integration neuer Medien in die Nutzungsmuster von Jugendlichen. In: Bug, Judith / Karmasin, Matthias (Hrsg.): Telekommunikation und Jugendkultur. Eine Einführung. Opladen, S. 29-46.

Hoffmann-Riem, Wolfgang / Schulz, Wolfgang / Held, Thorsten (2000): Konvergenz und Regulierung. Optionen für rechtliche Regelungen und Aufsichtsstrukturen im Bereich Information, Kommunikation und Medien. Baden-Baden.

Höflich, Joachim R. (1999): Der Mythos von umfassenden Medium. Anmerkungen zur Konvergenz aus einer Nutzerperspektive, In: Latzer, Michael et al (Hrsg.): Die Zukunft der Kommunikation, Phänomene und Trends in der Informationsgesellschaft. Innsbruck, Wien. S. 43-60.

Just, Natascha (2005): Competition Policy in Convergent Communication Sectors: Quo vadis? Konferenzbeitrag, 16th ITS - Regional Conference, Porto.

Latzer, Michael (1997): Mediamatik - Die Konvergenz von Telekommunikation, Computer und Rundfunk. Opladen.

Latzer, Michael (1998): Von der Medien- zur Mediamatik-Politik: Kommunikationspolitische Folgerungen aus dem Konvergenztrend. In: Österreichische Zeitschrift für Politikwissenschaft. 27(2), S. 143-156.

Latzer, Michael (2000): Toward an Integrated Universal Services Policy for the „Mediamatics“-Sector, in: Bohlin, Eric / Brodin, Karolina / Lundgren, Anders / Thorngeren, Bertil (Hrsg.): Convergence in Communications and Beyond, Amsterdam, S. 301-312.

Latzer, Michael / Just, Natascha / Saurwein, Florian / Slominski, Peter (2002): Selbst- und Ko-Regulierung im Mediamatiksektor. Wiebaden.

Latzer, Michael / Maier-Rabler, Ursula / Siegert, Gabriele / Steinmaurer, Thomas (Hrsg.) (1999): Die Zukunft der Kommunikation, Phänomene und Trends in der Informationsgesellschaft. Innsbruck, Wien.

Meier, Werner A. (1999): Was macht die Publizistik- und Kommunikationswisschenschaft mit der Konvergenz? In: Latzer, Michael et al (Hrsg.): Die Zukunft der Kommunikation, Phänomene und Trends in der Informationsgesellschaft. Innsbruck, Wien. S. 29-42

Murdock, Graham (2000): Digital futures: European Television in the age of convergence. In Wieten, J., Murdock, G., Dahlgren, P. (Hrsg.). Television Across Europe, A Comparative Introduction. London.

Napoli, Philip M. (2001): Foundations of Communications Policy, Principles and Process in the Regulation of Electronic Media. Hempton Press.

Napoli, Philip M. / Gillis, Nancy (2005): Reassessing the Potential Contribution of Communications Research to Communications Policy: The Case of Media Ownership. ICA Conference Paper. Boston.

Neverla, Irene (2001): Das Netz - eine Herausforderung für die Kommunikationswissenschaft. In: Maier-Rabler, Ursula / Latzer Michael (Hrsg.): Kommunikations- Kulturen zwischen Kontinuität und Wandel. Universelle Netzwerke für die Zivilgesellschaft. Konstanz, S. 29-48.

Noam, Eli (1993): Reconnecting Communications Studies with Communications Policy. In: Journal of Communication, 43 (3), S. 199-206.

OECD (1992): Telecommunications and Broadcasting. Convergence or Collision? Paris.

OECD (2003): The Implications of Convergence for Regulation of Electronic Communications, Report to the working Party on Telecommunication and Information Services Policies. Paris. 
OECD (2005): Telecommunication Regulatory Institutional Structures and Responsibilities, Report to the Working Party on Telecommunication and Information Services Policies. Paris.

Reeves, Byron / Baughman, James L. (2003) "Fraught with Such Great Possibilities": The Historical Relationship of Communication Research to Mass Media Regulation. In: Braman, Sandra (Hrsg.): Communication researchers and policy-making. Cambridge, S. 529-571.

Ronneberger, Franz (1992): Kommunikationspolitik, In: Burkart, Roland / Hömberg, Walter (Hrsg.): Kommunikationstheorien. Ein Textbuch zur Einführung. Wien, S.191-203.

Roßnagel, Alexander (Hrsg.) (2005): Neuordnung des Medienrechts. Neuer rechtlicher Rahmen für eine konvergente Technik? Baden-Baden.

Saxer, Ulrich (1999): Warnung vor großen medienpolitischen Windmaschinen. Plädoyer für eine wissenschaftliche Medienpolitik. In: Imhof, Kurt / Jarren, Otfried / Blum, Roger (Hrsg.): Steuerungs- und Regelungsprobleme in der Informationsgesellschaft. Opladen, S. 361-376.

Saxer, Ulrich (2006): Theorien oder nur Windmaschinen? Was hat das Fach in der medienpolitischen Forschung geleistet? Thesenpapier, IPMZ-Kolloquium: Ordnung durch Medienpolitik?, Zürich.

Scherer, Joachim (1985): Telekommunikationsrecht und Telekommunikationspolitik. BadenBaden.

Schulz, Wolfgang / Held, Thorsten (2004): Regulated Self-Regulation as a Form of Modern Government. Luton.

Simpson, Seamus (2004): Universal service issues in converging communications environments: the case of the UK. In: Telecommunications Policy, 28 (3/4), S. 233-248.

Verhoest (Hrsg.) (2005): Contradiction, Confusion and Hubris. A Critical Review of European Information Society Policy. ENCIP.

Wagner, Ulrike / Theunert, Helga / Gebel, Christa / Lauber, Achim (2004): Zwischen Vereinnahmung und Eigensinn - Konvergenz im Medienalltag Heranwachsender. München.

Windahl, Sven / McQuail, Denis (1993): Communication Models, 2.Auflage. London.

Wu, Irene (2004): Canada, South Korea, Netherlands and Sweden: regulatory implications of the convergence of telecommunications, broadcasting and Internet services. In: Telecommunications Policy, 28(1), S. 79-96. 